\title{
Falencias en la relación médico-paciente en la esclerosis múltiple
}

\section{| 1 Juan Pablo Lopera-Vásquez |}

Resumen: Una buena relación entre médicos y pacientes permite agilizar el diagnóstico de esclerosis múltiple y lograr adherencia a los tratamientos. Por eso, se buscó comprender las dificultades en la relación entre médicos y pacientes con esta condición de salud, a través de una investigación narrativa realizada en el Instituto Neurológico de Colombia de la ciudad de Medellín, en la que participaron cinco personas con esclerosis múltiple, dos de sus cuidadores y dos profesionales tratantes. Se realizaron entrevistas conversacionales a profundidad, se visitaron sus domicilios y se revisaron sus historias clínicas. Se construyó una narración polifónica para cada participante, de las que se extrajeron algunos relatos que revelan dificultades en la relación médico-paciente. Estos relatos evidencian falta de confianza hacia las propuestas terapéuticas de los médicos, así como expresiones, de parte de estos, que denotan menosprecio hacia sus pacientes. A esto se añade malestar por la brevedad de las consultas y dificultades para llegar al diagnóstico y para expresarlo apropiadamente. Esta situación revela dificultades para realizar un buen abordaje clínico, lo cual puede afectar los acuerdos entre médicos y pacientes, acerca de los mejores procedimientos terapéuticos a seguir.

> Palabras clave: esclerosis múltiple; relaciones médicopaciente; narración; confianza.
${ }^{1}$ Instituto Neurológico de

Colombia. Medellín, Colombia (juan.lopera@neurologico.org.co). ORCID: 0000-0001-6689-4834.
Recebido em: 17/01/2018 Revisado em: 12/06/2018 Aprovado em: 22/06/2018 


\section{Introducción}

La esclerosis múltiple es una condición autoinmune en la que se afecta el sistema nervioso de forma variada, lo cual deriva en síntomas poco precisos y que pueden desaparecer al menguar la inflamación de la mielina. Debido a eso, la fase previa al diagnóstico se caracteriza por la incertidumbre, que lleva a que las personas con esta condición de salud, así como sus familiares y amigos se cuestionen el carácter ilusorio de los síntomas. Solo cuando se presenta recurrencia y empeoramiento de estos, se buscan respuestas en los profesionales de la salud (KOOPMAN, SCHWEITZER, 1999).

El que los síntomas varíen, remitan al poco tiempo y no suelan tener respaldo en pruebas clínicas que los objetiven, genera riesgo de que la relación médicopaciente se altere desde la fase previa al diagnóstico, al no encontrar una explicación satisfactoria para los síntomas, con el consiguiente malestar para los pacientes y sus médicos. A esa situación se suma que quienes presentan afectaciones neurológicas, como la esclerosis múltiple, suelen consultar en las recaídas, justo cuando tienen dificultades para expresarse, lentitud en el habla y baja tono de voz, lo que genera problemas a los médicos, quienes les impiden explicar todo lo que les sucede, ya que suelen contar con un tiempo de consulta limitado (DUARTE-GARCÍA, 1999).

El no permitir expresar todo lo que les sucede o el interpretar erróneamente los síntomas inespecíficos, puede derivar en retrasos para establecer el diagnóstico y para comenzar los tratamientos. Además, los pacientes pueden sentir inconformidad al no poder expresar todos sus padecimientos, lo cual deteriora su relación con los médicos. Esto puede afectar nocivamente su salud, pues el establecer un vínculo adecuado entre médicos y pacientes, y la existencia de una buena comunicación entre ellos, conlleva beneficios para la salud psicológica y física del paciente, lo que aumenta su confianza y compromiso (BECK; DAUGHTRIDGE; SLOANE, 2002; LITTLE et al., 2001). Por el contrario, la mala comunicación puede alterar la confianza y la adherencia terapéutica.

Ante esa situación, en este estudio se buscó conocer las dificultades presentes en la relación entre médicos y pacientes con esclerosis múltiple, así como comprender cómo esas dificultades afectan la toma de decisiones acerca de tratamientos más apropiados para cada caso. 


\section{Método}

Se realizó una investigación narrativa llamada "Reconocimiento como potenciador de la autonomía y la calidad de vida: relato de un viaje alrededor de la esclerosis múltiple", en la que participaron cuatro mujeres y un hombre con esta enfermedad, dos de sus cuidadores y dos profesionales expertos en la patología. El primer participante se contactó en el servicio para enfermedades desmielinizantes del Instituto Neurológico de Colombia, en tanto que los siguientes se invitaron a través de metodología de bola de nieve, a partir de las comprensiones y dudas resultantes en el abordaje de los participantes previos. Esto fue posible en tanto esta población suele conocerse entre sí, al pertenecer a asociaciones de pacientes.

Con cada participante se sostuvo entrevistas conversacionales narrativas no estructuradas (CHASE, 2011), en las que se les solicitó relatar libremente su experiencia con la enfermedad a partir del pedido: "cuénteme su historia", lo cual implicó que no se tuvo una guía predefinida de preguntas ni de temas. Se visitó sus domicilios y se revisaron sus historias clínicas, con lo que se construyó una narración polifónica para cada uno. Por la polifonía (BAJTÍN, 1986; DUCROT, 1984) en cada narración aparecen las voces del participante y del investigador, y en algunas también se incluyen a personas cercanas al participante.

Se grabó la voz durante las entrevistas, las cuales fueron luego transcritas. El número de encuentros con cada participante, y su extensión, fue diferente con cada uno, de acuerdo a las particularidades que ellos querían incorporar en la narración de su experiencia. La construcción narrativa se basó en un ejercicio hermenéutico (GADAMER, 2006) de fusión del horizonte comprensivo de cada participante, con el horizonte comprensivo del investigador, quien se convirtió en un narrador de segundo orden al reconstruir sus relatos a través de la escritura. Esa fusión se apoyó en un ejercicio de reflexividad constante, que se registró en un diario de campo, el cual incluyó descripciones de lo sucedido en las entrevistas y permitió cuestionar las técnicas usadas (GUBER, 2001). Así mismo se cuestionaron y registraron los prejuicios, sensaciones y opiniones del investigador, así como los elementos a incluir en cada narración.

Para la construcción narrativa se tuvo en cuenta criterios de orden literario, cono son la verosimilitud, estética, reflexividad e impacto en el lector (RICHARDSON; ADAMS, 2005) y se siguió el círculo hermenéutico, de relacionar las partes con 
el todo (GADAMER, 2006). Para ganar en verosimilitud se describió el entorno observado en las visitas domiciliarias, en tanto eso enriquece las narraciones y le da un contexto espacial a la vida de cada participante. Además, se buscó que las narraciones correspondieran a lo que en efecto expresaron los participantes. Por eso, a cada uno se le entregó su respectiva narración para que la corrigiese o completase, lo cual permitió que el investigador y cada participante buscasen temas generadores (FREIRE, 2005) acerca de problemáticas presentes en sus encuentros clínicos, para construir conjuntamente soluciones o alternativas que las mejorasen.

En las narraciones se buscó revelar las experiencias de cada participante en su relación con la esclerosis, siendo los encuentros clínicos una parte importante de esa experiencia, los cuales impactan de forma diferente a cada individuo. Sin embargo, estas narraciones no hablan solo de sus experiencias específicas, pues cada participante mencionó vivencias de otros pacientes y profesionales de la salud que conocen, para poder explicar lo que les sucede a quienes presentan esta condición de salud. Además, al expresar sus experiencias se refirieron a contextos que les dan sentido. Por eso, en estas narraciones es posible analizar lo que puede suceder en la relación médico-paciente y en los contextos en que esta se presenta, como son las instituciones hospitalarias, el sistema de salud colombiano y las políticas que lo rigen, al igual que se pueden analizar elementos culturales y valores sociales que rodean a los participantes.

Para analizar esos asuntos se consideró el sentido que se aprecia en cada narración como una totalidad, aunque estas no aparecen en este artículo por cuestiones de espacio. Por eso, se decidió sustraer y analizar algunos relatos que reflejan formas de menosprecio en la interacción entre médicos y pacientes, pobreza en la confianza establecida, dificultades por la escasez de tiempo para las consultas, entre otros asuntos que denotan fallas en el ejercicio clínico. Para la selección de fragmentos no se utilizaron programas informáticos, que se basan en una lógica del pensamiento categorial poco coherente con el pensamiento narrativo que se privilegió en este estudio.

Se contó con el aval de los Comités de Ética de la Investigación de la Facultad Nacional de Salud Pública de la Universidad de Antioquia y del Instituto Neurológico de Colombia. Se siguieron las recomendaciones de la Resolución $\mathrm{n}^{\circ}$ 008430 de 1993, que establece las normas científicas, técnicas y administrativas para la investigación en salud en Colombia (MINSALUD, 1993). También se acataron los principios internacionales que protegen a quienes participan en investigaciones 
médicas según la Declaración de Helsinki y las pautas éticas internacionales para investigación biomédica en seres humanos (CIOMS, 2002). Igualmente se asumieron compromisos éticos de la investigación social, teniendo reciprocidad y protección a los participantes (GALEANO, 2003), en tanto que para revisar las historias clínicas se acató la Resolución número 1995 de 1999 del Ministerio de Salud (MINSALUD, 1999). Por último, para proteger la identidad de los participantes, en las narraciones se ficcionaron algunos asuntos sin afectar su verosimilitud.

\section{Resultados y discusión}

Ana, Sofía, Victoria, Jesús y Lucía son los pseudónimos de los participantes con esclerosis múltiple, en tanto que Luisa y Martín son los de los cuidadores. El neurólogo y la médica general aparecen en las narraciones sin nombre ni seudónimo al no lograr establecer una identidad narrativa para ellos. Los cinco participantes con esclerosis múltiple oscilan entre los cuarenta y sesenta años de edad. Una estudió artes plásticas, otra se jubiló luego de trabajar como secretaria, otra tiene una papelería en su casa, la otra es ama de casa y el hombre trabaja en un parqueadero. Todos son bachilleres y tres de los cinco cuentan con escasos ingresos económicos. Todos presentan esta condición de salud desde hace más de veinte años, la cual les comenzó a principios de la tercera década de la vida. En consecuencia, en sus narraciones mencionaron lo vivenciado con múltiples profesionales de la salud que los han atendido durante ese tiempo. Por su parte, el neurólogo y la médica general son expertos en el manejo de esta patología, por lo que cuentan con conocimiento amplio de las problemáticas que estos pacientes encuentran al consultar al sistema de salud.

\section{Síntomas iniciales y diagnóstico}

El diagnóstico se presentó de forma tardía en tres de estas personas. Todos los participantes refirieron que ese retraso, tanto en sus casos como en el de otras personas que conocen, se asoció a la inespecificidad de los síntomas y al poco conocimiento de esta entidad por parte de los médicos. Esto último se aprecia en la historia de Jesús, quien se tardó más de veinte años en ser diagnosticado. Al respecto mencionó:

Las caídas comenzaron en los años ochenta... Para esa época la esclerosis no se tenía en cuenta en el trópico, pues hay pocos casos y las clínicas no estaban preparadas para su diagnóstico. 
Ese pobre conocimiento médico se evidenció también en el caso de Victoria, quien contaba con una resonancia en la que se sugería una posible esclerosis múltiple, pero pasaron cinco años hasta que un neurólogo le explicó en qué consistía ésta, ya que quienes la atendieron en ese tiempo no sabían a qué se refería esa posibilidad diagnóstica.

El escaso conocimiento de los médicos acerca de la esclerosis se debe a que esta poco se aborda durante el proceso educativo, tal como lo expresó la médica entrevistada. Dice ella: "por su poca prevalencia en Colombia, mi contacto con esta patología se reducía a una clase en la universidad”. En efecto, por mucho tiempo se creyó que la esclerosis no se presentaba en países tropicales, lo cual derivó en que se enseñara poco acerca de ella, considerándose que afectaba solo a países septentrionales. Por eso, únicamente Ana y Sofía obtuvieran un diagnóstico temprano, ya que Ana es de ascendencia europea y Sofía es norteamericana. Pero en el caso de los otros participantes, ningún médico consideró, de forma temprana, un posible diagnóstico de esclerosis múltiple.

La falta de conocimiento no solo deriva en demoras para diagnosticarla, también lleva a diagnósticos y tratamientos erróneos, como le sucedió a Jesús, a quien le diagnosticaron tendinitis benigna y luego mielopatía de cola de caballo. En su caso, la dificultad para establecer un diagnóstico se debió también a que, por la pérdida de empleos, debía cambiar de aseguradora, lo cual derivaba en cambios frecuentes del profesional tratante. Con cada cambio, el nuevo médico debía actualizarse acerca de una historia clínica cada vez más grande y confusa, sin lograr establecer un diagnóstico.

Este cambio constante de profesional tratante fue un malestar generalizado en los participantes, incluyendo los profesionales tratantes, quienes refirieron dificultades para hacer seguimiento a los pacientes. Esos continuos cambios se debieron principalmente a condiciones del sistema de salud colombiano. Este se encuentra constituido por empresas aseguradoras del riesgo en salud, que contratan servicios con las clínicas, públicas o privadas. Cuando los usuarios del sistema cuentan con empleo aportan económicamente a este, pero cuando lo pierden pasan a ser subsidiados por el estado. Esto implica que los cambios de empleo, o su pérdida, derive en cambios de aseguradora. Tal situación, así como las frecuentes variaciones en las contrataciones entre las aseguradoras en salud y las entidades hospitalarias, genera cambios de lugar de atención a los pacientes. 
Otra característica de la fase previa al diagnóstico es la desaparición de los síntomas, lo cual puede interpretarse como un rasgo de cuadros psiquiátricos. Cuando estos se plantean como posibilidades diagnósticas, quienes presentan esclerosis se sienten incomprendidas. Al respecto, Victoria expresó:

"A mi mamá le dijeron que yo estaba loca... Salí medicada con fluoxetina, clonazepam y otra droga para locos. Mi mamá le dijo a mi padrastro que tuviera cuidado porque estaba loca, y mi hermanita me contó: mi hermano y mi mamá dicen que usted se hace, que no está enferma.

Igualmente, Lucía, a quien la clarificación diagnóstica le tardó diez años, dijo:

Consultaba y me decían cosas diferentes, incluso me tildaron de hipocondriaca... Me mandaban carbamacepina, calmantes como para locos y vitaminas. Pese a que insistía que me remitieran a neurología, los médicos decían que no lo requería.

Estas situaciones incomodan a estas personas y las hace sentir menospreciadas, lo cual afecta la relación con sus médicos. Eso puede deberse a que esa respuesta médica se relaciona con la legitimación o no que hagan de sus síntomas, lo cual se ha demostrado que tiene efecto en la identidad de las personas y en su sufrimiento (CANESQUI, 2018).

Ante tal panorama, al llegar al diagnóstico de esclerosis múltiple se esperaría que mejorara la relación, al acabar con la incertidumbre. Sin embargo, al determinarse el diagnóstico no hay mejoría en la relación médico-paciente, lo cual puede deberse a que en ese momento se presenta falta de tacto y de compasión, tal como lo refirió Ana:

Cuando me diagnosticaron me dijo el neurólogo: tiene que cambiar de vida. Ante esto sentí que ¡hubiera querido morirme!, pues enseñaba gimnasia y danza, mi mundo era ser bailarina. Pero él me dijo: haga otra cosa. Era como si me hubiera dicho se puede morir mañana. ¡Es lo que he hecho toda mi vida! ¿Cómo me va a decir que no haga nada? ... No puede volver a hacer lo que hace, porque pierde el equilibrio y quizás dentro de ocho días o en un año sea inválida, siguió diciéndome el neurólogo. ¡Me mató, me mató!

Victoria también expresó que el recibir el diagnóstico fue poco grato:

A los cinco años de estar enferma, de ir y venir, me remitieron de nuevo al neurólogo, que
vio los exámenes y me dijo: tiene esclerosis múltiple, es muy horrible y no tiene cura”. ¿ $\mathrm{Me}$
voy a morir?, le pregunté. No, pero el tratamiento es costoso. Se va a quedar en la cama y en
silla de ruedas, me contestó. Yo pensaba: ;ya estoy muerta! ¡Lloré demasiado!

La falta de tacto para brindar el diagnóstico no se refiere solo a que el médico mencione las dificultades asociadas a la esclerosis, también a que ignore las particularidades de cada persona. En el caso de Jesús se aprecia que el neurólogo, 
pese a que le señala las dificultades que podrán presentarse a futuro, sí consideró su historia particular, así como su necesidad de escuchar una apreciación contundente respecto al diagnóstico, acerca del cual Jesús mencionó:

Este llegó en el 2005 cuando pidieron resonancia magnética, fue una pelea conseguirla y mi tía envió dinero para hacérmela particular. El neurólogo dijo que era esclerosis múltiple y me explicó que no tenía cura, que estaría con bastón, caminador, silla de ruedas y finalmente en la cama. Me mató con eso, pero quería que no ocultara nada. Dijo todo tal cual, el diagnóstico y la proyección, así debió ser. Le agradecí y estreché la mano, él era sorprendido.

El caso de Jesús muestra que es importante, al diagnosticar, conocer las vivencias particulares y el nivel de preparación de cada persona para hablar acerca del diagnóstico y del pronóstico. $\mathrm{Al}$ respecto se ha encontrado que para quienes el período sintomático previo al diagnóstico fue largo y complejo, el ser diagnosticados constituye un alivio (ISAKSSON; AHLSTRÖM, 2006). En efecto, eso fue lo que sucedió con Jesús, pese al terrible pronóstico planteado.

Con la incertidumbre que generan los síntomas, en estudios previos también se ha reportado que las personas conectan los síntomas en una conjunción explicativa en la que concluyen que tienen un infarto cerebral, que se van volver ciegos o que se están enloqueciendo (BARKER-COLLO; CARTWRIGHT; READ, 2006). Por eso, se ha evidenciado que hay personas para quienes el diagnóstico les disminuyó la ansiedad, al descubrir que no tienen un cuadro que represente una muerte inminente (MUSHLIN et al., 1994).

Por su parte, otras personas mostraron ambivalencia, pues además de miedo, malestar y pensamientos acerca de la muerte o de quedar confinados a una silla de ruedas, incrementaron su habilidad para tolerar y controlar la esclerosis, a partir de información positiva que encontraron acerca de esta. Así mismo, hay quienes a partir de ese momento desarrollaron estrategias para manejar síntomas y limitaciones (DENNISON et al., 2010).

Estas situaciones develan que el diagnóstico es un momento de respuestas, rupturas e inauguración de asuntos en la vida de estas personas. Para unos importa que brinde respuestas, para otros representa la posibilidad de elaborar estrategias para manejar síntomas, y hay otros para quienes es una tragedia, al romper con lo que preveían para sus vidas. Esto último se aprecia en un estudio narrativo en el que se muestra cómo se modificó la historia de vida de una mujer felizmente casada, 
madre de dos hijos, que creía haber alcanzado todo en su vida; pero el diagnóstico le amenazó su identidad, proyectos y relaciones con los demás (FINLAY, 2003).

Volviendo a los participantes en este estudio, se aprecia que para Ana, el saber que la esclerosis la afectaría en dimensiones tan valiosas representó una ruptura en su biografía personal, mientras que para Jesús el diagnóstico fue la culminación de años de incertidumbre, lo cual derivó en que viviesen el diagnóstico de forma diferente. Esas rupturas biográficas se presentan en general, y de formas distintas, en las condiciones de salud que implican diferentes dolencias crónicas que inician a lo largo de la vida (BURY, 2011) e incluso en aquellas que comienzan desde la infancia (CASTELLANOS; BARROS; COELHO, 2018). Tal situación indica que los profesionales de la salud deberían conocer las singularidades de cada persona, para comprender cómo podría darse esa ruptura biográfica y poder explicar el diagnóstico apropiadamente, además de prever cómo podrían asumir el pronóstico y los tratamientos.

Ese conocimiento debe acompañarse de autocuestionamientos acerca de la información que es pertinente brindar acerca de la enfermedad. Al respecto, en las narraciones de la médica general y del neurólogo se evidenció que se inquietan acerca de esto, mostrando cuidado hacia sus pacientes. Sin embargo, sus historias denotan que desconocen cuánta información deben darles, pese a sus esfuerzos autoformativos para aprender qué es lo apropiado para informar a cada persona.

Por su parte, el médico que diagnosticó a Lucía, y la médica que hizo lo propio con Victoria, se mostraron afligidos con el diagnóstico, evidenciado su sensibilidad, pero sin saber cómo expresarse. Por el contrario, el de Jesús pareció cruel, al señalarle un pronóstico sombrío. Pese a eso, también hizo recomendaciones, en las que sugiere analizar su singularidad, al mencionarle una asociación de pacientes. Al respecto le dijo:

Allá se ven casos muy tristes, podría afectarlo. Es mejor abstenerse, lo mismo que del
internet, no llenarse de paranoia e información equivocada. Haga lo que quiera, pero
puede ser bueno o malo.

Estas advertencias, acerca de cuidarse de la información disponible en internet, y acerca de conocerse bien, para saber si es pertinente contactar a las asociaciones, denotan reflexión respecto a las incertidumbres presentes al momento de diagnosticar. Incertidumbres que son grandes en los médicos, al desconocer cómo es la vida de sus 
pacientes. El desconocimiento de las particularidades existenciales de estos puede ser una de las razones por las que personas con esclerosis múltiple se quejan del poco apoyo y de la escasa información que reciben de los médicos al ser diagnosticadas (IRVINE et al., 2009). Incluso, hay quienes se enojan por la forma en que reciben el diagnóstico y hacen sus propias búsquedas de información para aprender sobre la enfermedad (EDWARDS; BARLOW; TURNER, 2008).

\section{Menosprecio, consultas breves y desconfianza}

Cuando finalmente se diagnostica la esclerosis múltiple, se esperaría que mejore el manejo de la problemática y la relación médico-paciente, al contar con una explicación sólida para los síntomas y recaídas. Sin embargo, los relatos de los participantes evidenciaron que eso no suele suceder, al presentarse prácticas de menosprecio. Las narraciones muestran que se presentan intervenciones médicas que develan incomprensión e intolerancia de parte del personal de salud. Eso lo vivió Victoria, quien expresó:

En una de las últimas recaídas me quedé sin voz y la doctora dijo que si no le hablaba, no me atendía.

También le sucedió que, previo al diagnóstico, otro médico hizo una interpretación irrespetuosa de su situación. Al respecto refirió:

Los médicos decían que era estrés, problemas de circulación y otras cosas. Incluso uno dijo que yo necesitaba un hombre.

Lucía también relató una situación en la que se revela insensibilidad por parte del personal de salud, al consultar en una clínica que no le correspondía. Lucía expresó:

Busqué atención cerca a la casa pues los pies no me funcionaban. Me prestaron silla de ruedas y unos caballeros me bajaron porque no había ascensor. La doctora dijo, al ver mi cédula, ella consulta en el centro, tiene que estar boqueando para atenderla.

Ante este tipo de situaciones, Lucía considera que los médicos son muy inhumanos, por lo que se sorprendió al encontrar uno que fue muy cálido en un momento en que ella estaba muy decaída. Al respecto, Lucía dijo:

En la casa soy fuerte, pero ¿puedo llorar? El médico me abrazó y dijo: Dios nos da inteligencia y sabiduría, sin él no hacemos nada, a él lo encontrarás en cualquier parte y a la hora que sea. ¡Decirme eso un médico, cuando se vuelven tan inhumanos!, lo sentí tan afín. Eran mensajes de vida y fortaleza. Entonces le dije: la próxima vendré caminando. 
Estas prácticas de menosprecio que estas personas experimentan en los encuentros clínicos, no se limitan a la relación con sus médicos, pues Ana relató que también ha sucedido con fisioterapeutas:

Me ponían compresas calientes, después heladas y masajes. Eso no es para un problema neurológico. Creen que todo es de ortopedia. ¿Cómo se le ocurre?, me hace daño, le decía a la fisioterapeuta. Ella me contestaba: asi se usa aqui. Al final se enfureció y me dijo: no la atiendo más. A la tercera cita dejé de asistir. Es ofuscador, hace más daño eso que no hacer nada. Además, son ofensivos, pues me decía: usted cree que sabe mucho, pero tiene que ponerse las compresas. Son cerradas, tienen un entrenamiento creyendo que todo el mundo es igual. Yo salía furiosa.

\section{Algo similar le sucedió en hidroterapia:}

Hay un montón de personas con parálisis cerebral, entonces para el hidroterapeuta todos tenemos eso. Pedí que no nos igualaran, le explicaba y me daba rabia.

Estos relatos develan que las prácticas de menosprecio están presentes en las atenciones clínicas y de rehabilitación. En particular, parece menospreciarse el saber de cada persona acerca de su condición de salud, de sus dolencias y de las formas en que las maneja. Ese menosprecio puede ser una de las razones por las que se ha encontrado que en la esclerosis múltiple, los médicos y pacientes asumen su relación como un enfrentamiento (TOOMBS, 1992). A eso se ańade que la carencia de tiempo en las consultas sigue afectando cuando ya se estableció un diagnóstico. Al respecto, el neurólogo señaló:

La gran falencia es el poco tiempo que se les dedica. El asunto debiera ser espontáneo, explicarles estructuradamente la enfermedad, escuchar tratamientos alternativos, explicar si tienen evidencia para nuestra medicina o si pueden ser peligrosos. Debiera hacerse una charla estructurada o conversatorio sobre todos los tópicos para mejorar la confianza para hablar sobre algunos temas. Por ejemplo, la sexualidad ni se aborda o se hace dentro de una lista de chequeo y revisión por sistemas, en la que se pregunta por el pensamiento, memoria, visión, problemas para respirar, moverse, dormir y para la vida sexual. Eso es muy estructurado, no alcanzan a procesar qué se preguntó.

Esa escasez de tiempo lleva a que estas personas perciban poco interés de parte de sus médicos, aunque los relatos sugieren que no todo se explica por lo limitado del tiempo, sino que en efecto los médicos podrían mostrar desinterés y malestar hacia estas personas. Eso se aprecia en lo que afirmó la médica general acerca de lo que le dijeron al contratarla en una institución para manejar a esta población:

Me encomendaron los pacientes con esclerosis múltiple, que a su modo de ver eran complejos por la patología y por su forma de ser. Les parecían susceptibles y caprichosos, pues se enojaban fácilmente y no asistían a consulta, nadie quería encargarse de ellos. 
Esta médica, al conocer mejor a estas personas encontró que esas complejidades tenían que ver con dificultades para desplazarse a los sitios de consulta, malestares que genera el hacer filas en las clínicas y el tener que esperar demasiado por las atenciones médicas, las cuales son tan cortas que impiden expresar toda su problemática.

Dada la brevedad de las consultas y los comentarios que denotan menosprecio, no es extraño que estas personas desconfíen del criterio médico, como se evidencia en las palabras de Lucía, quien expresó desconfiar de las propuestas acerca de iniciar o cambiar el tratamiento. Lucía dijo: "Creo que los laboratorios les ofrecen algo, pues ellos son buscando qué candidatos sirven”. Victoria también refirió su desconfianza, al expresar:

Otro neurólogo siempre me veía grave, decía: es una enfermedad progresiva y está muy avanzada. Iba allá y era como si tuviera la lápida colgada. Este me propuso ensayar un nuevo medicamento. Le respondí que no quería y que los neurólogos ganan algo mandando medicamentos. No ensayé por los efectos secundarios, podía darme algo en el cerebro que me dejaría como un vegetal, además podía afectar el corazón. Le contesté así porque ellos insisten e insisten sin uno querer, pero no es pensando en que uno se alivie, ¡eso son mentiras!

Los médicos tienen algo de consciencia de que esta desconfianza puede existir, pese a que no siempre los pacientes la expresan. Al respecto, el neurólogo refirió:

En estas enfermedades con medicamentos complejos, los programas de pacientes son la moda, el plus de la industria farmacéutica. Al enterarse que eso lo paga la casa productora del medicamento, podrían pensar que hay un trato con el médico y que debido a eso lo manda, pero jamás me expresan ese cuestionamiento.

Estos relatos relevan que en la relación médico-paciente, en la esclerosis múltiple, pueden presentarse falencias importantes y formas de menosprecio, en particular al valorar las singularidades de la vida de cada paciente. Esto se relaciona con la focalización del personal de salud en aspectos biomédicos de la enfermedad, lo cual se aprecia en las historias clínicas de estas personas, en las que se encontró que solo se consignan datos de orden biomédico, como son los síntomas, recaídas, disfuncionalidades y tratamientos farmacológicos, sin mencionar problemáticas de orden subjetivo, social, familiar o en la obtención de servicios requeridos para el manejo de la enfermedad.

En la esclerosis múltiple el abordaje biomédico ha permitido clarificar vías inmunológicas y genes de riesgo involucrados, brindó pistas sobre factores desencadenantes ambientales, mejoró el seguimiento a través de resonancia magnética, 
y generó más terapias y con mayor eficacia (HAUSER; CHAN; OKSENGERG, 2013). Sin embargo, eso no debería derivar en que en el ejercicio clínico se ignoren otros aspectos de la enfermedad, pues eso lleva a limitar la idea de lo que es la salud y puede afectar la relación entre el personal de salud, pacientes y cuidadores.

Esta focalización en lo biomédico también se debe a que los mismos pacientes omiten información que no encuadra en esa mirada. Al respecto, el neurólogo dijo que estos callan asuntos por no considerarlos científicos y porque los asumen como poco pertinentes para la consulta. Eso puede deberse a que estas personas, al igual que sus médicos, asumen las instituciones de salud, como espacios solo para abordar asuntos biomédicos. Si los actores presentes en una cita médica consideran que allí únicamente deben hablar del organismo, y no de su vida en general, solo expresarán lo que encuadre con esa expectativa. Eso condiciona el encuentro clínico, el cual también se limita por las exigencias contextuales de la vida moderna y del sistema de salud colombiano.

\section{Contexto de la relación clínica en la actualidad}

Una de esas limitantes es el poco tiempo de consulta. Al respecto, el neurólogo mencionó que este suele oscilar entre veinte y treinta minutos, lo cual no alcanza para examinar al paciente, llenar su historia clínica y revisar el largo expediente de exámenes que suelen acumular después de cada recaída. El que las citas médicas sean cortas es una exigencia de la modernidad, que se debe a una racionalidad económica en la que predomina la búsqueda de sostenibilidad del sistema de salud y la rentabilidad.

Otra exigencia que recae sobre la relación médico-paciente es que esta pasó de un modelo paternalista a uno autonomista, en el que cada individuo exige conocer todo lo relacionado con su problemática (MCCOY, 2008). Por eso, cuando los pacientes son excluidos de las decisiones clínicas se sienten tratados como objetos y pierden confianza en el profesional (COSTA-ALCARAZ et al., 2011). El enfermo dejó de ser un receptor pasivo para considerarse agente con derechos y capacidad de decisión sobre procedimientos diagnósticos y terapéuticos, mientras que el médico es ahora un asesor que ofrece conocimientos y consejos. En consecuencia, la relación clínica pasó de ser bipolar, vertical e infantilizante, a horizontalizarse y colectivizarse, al contar también con la participación de múltiples profesionales sanitarios (LÁZARO; GRACIA, 2006). Eso se ha asociado a que los pacientes hoy acceden a más información 
acerca de sus enfermedades, lo que lleva a convertirse con frecuencia en "pacientes expertos”, lo cual genera antipatía en muchos médicos (SHAW; BAKER, 2004).

A esto se suma que en la actualidad se subvalora el ejercicio clínico, en tanto requiere tiempo, no genera grandes ingresos y se considera equivocadamente que la tecnología puede sustituirlo (MORENO, 1998). Además, el encuentro clínico incorporó la "medicina basada en la evidencia", que se refiere al uso consciente, explícito y juicioso de la evidencia científica disponible para decidir intervenciones (VEGA-DE CÉNIGA et al., 2009). Esa perspectiva entró en conflicto con la "medicina basada en el paciente". Mientras la primera se ancla conceptualmente en la investigación, la segunda lo hace en el cuidado médico (SACRISTÁN, 2013).

Esta situación se ve reflejada en las palabras de Sofía, quien expresó que el corto tiempo de consulta se articula con el poco interés que muestran los profesionales de la salud. Al respecto mencionó lo que se sucedió con dos médicos:

Hubo uno que solo me renovaba el medicamento, nunca encontré interés de su parte... Hubo otro que durante toda la cita escribió en el computador lo que decían los exámenes. Quedé como si me tiraran un balde de agua fría, no se esforzó por intercambiar ideas.

El revisar los resultados de exámenes, en vez escuchar al paciente, es una muestra de cómo se privilegia la evidencia científica, sobre los relatos que los pacientes pueden expresar acerca de lo que sucede en su cotidianidad. Eso da indicios de que la relación médico-paciente se ha tecnologizado y cientifizado, lo cual deriva en que el profesional tratante esté más atento a sus conocimientos científicos y a los resultados de exámenes derivados de las nuevas tecnologías, que a lo expresado por el paciente. A este ya no lo ven como un humano concreto con dilemas particulares, sino como el correlato de lo expresado en los exámenes y en la literatura médica. Es decir, el paciente no es reconocido en sus particularidades. Esa actitud incómoda a los consultantes y deriva en resentimientos y desconfianza hacia los médicos, con el consiguiente deterioro de la relación, lo cual afecta la adherencia. De hecho, para conseguir buena adherencia terapéutica se requiere un buen vinculo y que el profesional sepa qué hacer, cómo hacer y cuándo hacer (LIBERTAD, 2004).

Esta situación se enmarca en el hecho de que en Colombia el derecho a la salud se construyó en la mentalidad de las personas como una caridad obtenida por su situación de miseria, como un servicio según la capacidad de pago individual o como prestación social por acceder a un trabajo; pero no como una verdadera garantía estatal (HERNÁNDEZ, 2000). Además, en el país prima la libertad económica 
sobre la racionalidad social del derecho a la salud, lo cual derivó en la implementación de la Ley 100 de 1993, que definió el sistema de salud (GAÑAN, 2013). Con esta ley se incorporaron las sugerencias del Banco Mundial, acerca de invertir en salud, integrando inversionistas privados para obtener competitividad en este mercado, lo que mejoraría la prestación de los servicios (BANCO MUNDIAL, 1993).

Esta preeminencia de la racionalidad económica se refleja en que las sucesivas transformaciones del sistema de salud, durante dos décadas, no han modificado la lógica mercantil de base (URIBE, 2011). Además, esa racionalidad ha limitado el tiempo de las consultas, lo cual deriva en que el médico no logre conocer bien la condición de salud y la vida de cada paciente. Igualmente, acarrea cambios de hospitales para las atenciones y de médicos tratantes, y genera multiplicidad de exámenes y hospitalizaciones, que se desprenden de recaídas innecesarias, las cuales se presentan debido a que la falta de confianza en los médicos lleva al abandono de los tratamientos.

\section{Consideraciones finales}

La esclerosis múltiple es una patología del campo neurológico. Por tanto, que no sea suficientemente conocida por los neurólogos en Colombia, debido a su poca prevalencia, refleja que en la academia se menosprecia la importancia de estudiar lo que es poco frecuente en la población. Eso indica que para la formación médica sólo importa lo prevalente, que equivale a descuidar a las minorías con patologías extrañas, lo cual debe cuestionarse en la salud pública y en los ejercicios clínicos, pues el interés no debe girar solo en torno a lo más prevalente.

Por su parte, no es adecuado que los profesionales de la salud privilegien la mirada biomédica e ignoren las particulares de los pacientes. La esclerosis múltiple es una condición que se explica, en gran medida, por un orden biomédico. Pero este no logra explicar todos los malestares de los pacientes. Por eso, es necesario que la perspectiva biomédica no predomine, sino que se articule con otras perspectivas de la enfermedad, en las que se aborden los asuntos subjetivos, sociales, culturales, económicos y políticos, que afectan la prestación de servicios de salud y las formas de enfrentar y asumir la enfermedad.

Para dar cuenta de esos asuntos es necesario conocer la historia de vida de cada persona. Precisamente, esas historias en este estudio muestran que para entender el cambio existencial de las personas con esclerosis múltiple hay que trascender 
el enfoque biomédico, el cual no alcanza a explicar todo lo que les afecta. Para lograr una buena atención clínica se debe indagar por sus experiencias y dificultades particulares, pues al pasar el tiempo, las experiencias se articulan biográficamente de formas diferentes en cada individuo e influyen de maneras distintas en la calidad de vida (COLINET, 2013).

Finalmente, este estudio muestra que es necesario abandonar la racionalidad económica y pragmatista que predomina en las atenciones clínicas. Esa racionalidad afecta la relación médico-paciente, lo cual deriva en dificultades para establecer diagnósticos acertados, así como para hacer buenas elecciones de los tratamientos, lo que al final puede representar mayores costos económicos para el sistema de salud, así como un impacto negativo en la vida de las personas con esclerosis múltiple.

\section{Agradecimientos}

A los participantes de la investigación "Reconocimiento como potenciador de la autonomía y la calidad de vida: relato de un viaje alrededor de la esclerosis múltiple", que relataron sus historias de vida; al Departamento Administrativo de Ciencia, Tecnología e Innovación de Colombia (COLCIENCIAS), que patrocinó la formación doctoral del autor; y al Centro de Esclerosis Múltiple y Enfermedades Desmielinizantes (CEMED) del Instituto Neurológico de Colombia, que apoyó la realización del estudio y el abordaje de los participantes.

\section{Referencias}

BAJTÍN, M. Problemas de la poética de Dostoievski. México: Fondo de Cultura Económica, 1986.

BANCO MUNDIAL. Informe sobre el Desarrollo Mundial. Invertir en salud (Resumen). Washington DC: Banco Mundial, 1993.

BARKER-COLLO, S.; CARTWRIGHT, C.; READ, J. Into the Unknown: The Experiences of Individuals Living with Multiple Sclerosis. Journal of Neuroscience Nursing., v.38, n.6, p.435446, 2006.

BECK, R. S.; DAUGHTRIDGE, R.; SLOANE, P. D. Physician-Patient Communication in the Primary Care Office: A Systematic Review. The Journal of the American Board of Family Practice, v. 15, n. 1, p. 25-38, 2002.

BURY, M. Doença crônica como ruptura biográfica. Revista Tempus Actas de Saúde Coletiva, v. 5, n .2, p. 41-55, 2011. 
CANESQUI, A.M. Legitimidade e náo legitimidade das experiências dos sofrimentos e adoecimentos de longa duração. Ciência \& Saúde Coletiva., v. 23, n. 2, p. 409-416, 2018.

CASTELLANOS, M.; BARROS, N.; COELHO, S. Rupturas e continuidades biográficas nas experiências e trajetórias familiares de crianças com fibrose cística. Ciênc. saúde coletiva, v. 23, n. 2, p. 357-368, 2018.

CHASE, S. Narrative Inquiry. Still a Field in the Making. En N. DENZIN, N.; LINCOLN, I. (Eds.), The SAGE Handbook of Qualitative Research. Thousand Oaks: SAGE Publications., p. 421-434, 2018.

CIOMS \& ORGANIZACIÓN MUNDIAL DE LA SALUD. Pautas éticas internacionales para la investigación biomédica en seres humanos. Ginebra: OMS, 2002.

COLINET, S. La "carrera" de las personas que sufren de esclerosis múltiple: tres momentos clave. De la irrupción de la enfermedad al "trabajo biográfico". Revista Española de Investigaciones Sociológicas., v. 144, n. 1, p. 127-138, 2013.

COSTA-ALCARAZ, A. M. et al. Reconocimiento recíproco y toma de decisiones compartida con el paciente. Revista Clínica Española., v. 211, n. 11, p. 581-586, 2011.

DENNISON, L. et 1. Experiences of Adjusting to Early Stage Multiple Sclerosis. Journal of Health Psychology, v. 16, n. 3, p. 478-488, 2010.

DUARTE-GARCÍA, J. La relación médico-paciente en las enfermedades neurológicas. Revista de Neurología, v. 29, n. 7, p. 642-647, 1999.

DUCROT, O. El decir y lo dicho. Buenos Aires, Argentina: Hachette, 1984.

FINLAY, L. The Intertwining of Body, Self and World: A Phenomenological Study of Living with Recently-diagnosed Multiple Sclerosis. Journal of Phenomenological Psychology., v. 34, n. 2, p. 157-178, 2003.

FREIRE, P. Pedagogía del oprimido. México: Siglo XXI Editores, 2005.

GADAMER, H. G. Verdady Método II (Séptima). Salamanca, España: Ediciones Sígueme, 2006.

GALEANO, M. Seminario internacional "Ética en investigación social y educativa": Conclusiones y líneas de trabajo $\mathrm{N}^{\circ}$ 22. En Cuadernos Pedagógicos. Medellín, Colombia: Universidad de Antioquia, p. 143-53, 2003.

GAÑAN, J. L. Los muertos de la Ley 100. Prevalencia de la libertad económica sobre el derecho fundamental a la salud: una razón de su ineficiencia. Caso del Plan Obligatorio de salud del Régimen Contributivo. Medellín, Colombia: Editorial Universidad de Antioquia, 2013.

GUBER, S. La etnografía: método, campo y reflexividad. Bogotá, Colombia: Grupo Editorial Norma, 2001. 
HAUSER, S. L.; CHAN, J. R; OKSENGERG, J. R. Multiple Sclerosis: Prospects and Promise. Annals of Neurology, v. 74, n. 3, p. 317-327, 2013.

HERNÁNDEZ, M. El derecho a la salud en Colombia: obstáculos estructurales para su realización. Rev. Salud pública, v. 2, n. 2, p. 121-144, 2000.

KOOPMAN, W.; SCHWEITZER, A. The Journey to Multiple Sclerosis: A Qualitative Study. Journal of Neuroscience Nursing, v. 31, n. 1, p. 17-26, 1999.

LÁZARO, J.; GRACIA, D. La relación médico-enfermo a través de la historia. Anales del Sistema Sanitario de Navarra, v. 29, n. 1, p. 7-17, 2006.

LIBERTAD, M. A. Acerca del concepto de adherencia terapéutica. Rev Cubana Salud Pública, v. 30, n. 4, p. 1-5, 2004.

LITTLE, P. et al. Observational study of effect of patient centredness and positive approach on outcomes of general practice consultations. BMJ, v. 323, n. 7318, p. 908-911, 2001.

MCCOY, M. Autonomy, consent, and medical paternalism: legal issues in medical intervention. J Altern Complement Med., v. 14, n. 6, p. 785-792, 2008.

MINSALUD. Resolución 8430 de 1993. Octubre 4. República de Colombia: Ministerio de Salud, 1993.

MINSALUD. Resolución número 1995 de 1999. Julio 8. República de Colombia: Ministerio de Salud, 1999.

MORENO, M. A. Crisis del método clínico. Revista Cubana de Medicina, v.37, n.2, p.123128, 1998.

MUSHLIN, A. et al. The Value of Diagnostic Information to Patients with Suspected Multiple Sclerosis. Rochester-Toronto MRI Study Group. Arch Neurol., v. 51, n. 1, p. 67-72, 1994.

RICHARDSON, L.; ADAMS, E. Writing A Method of Inquiry. En DENZIN, N.; LINCOLN, I. (Eds.), Handbook of Qualitative Research (Third Edition). SAGE Publications., p.959-978, 2005.

SACRISTÁN, J. A. Medicina basada en la evidencia y medicina centrada en el paciente: algunas reflexiones sobre su integración. Revista Clínica Española., v. 213, n. 9, p. 460-464, 2013.

SHAW, J.; BAKER, M. “Expert patient”:dream or nightmare? BMJ, v. 328, p. 723, 2004.

TOOMBS, S. K. The Meaning of Illness: A Phenomenological Account of the Different Perspectives of Physician and Patient. Dordrecht, Países Bajos: Kluwer Academic Publishers, 1992.

URIBE, M. Los vaivenes de las politicas sociales en Argentina, Colombia, Chile, México y Uruguay ¿Neo o posneoliberalismo? México: Editorial Porrúa, 2011.

VEGA-DE CÉNIGA, M. et al. Medicina basada en la evidencia: concepto y aplicación. ANGIOLOGIA, v. 61, n. 1, p. 29-34, 2009. 


\section{Abstract}

\section{Flaws in the doctor-patient relationship in multiple sclerosis}

A good relationship between doctors and patients makes it possible to speed up the diagnosis of multiple sclerosis and achieve adherence to treatments. So we tried to understand the difficulties in the relationship between doctors and patients with this health condition, through a narrative research conducted at the Neurological Institute of Colombia in the city of Medellin, in which five people with multiple sclerosis, two of their caregivers and two treating professionals participated. Conversational interviews were conducted in depth, their homes were visited and their clinical histories were reviewed. A polyphonic narration was constructed for each participant, from which some stories that reveal difficulties in the doctor-patient relationship were extracted. These stories show a lack of confidence in the therapeutic proposals of the doctors, as well as the expressions from them, that denote contempt towards their patients. Discomfort due to the shortness of the consultations and difficulties to reach the diagnosis and to express it properly is added. This situation reveals difficulties to perform a good clinical approach, which may affect the agreements between doctors and patients, about the best therapeutic procedures to follow.

Keywords: multiple sclerosis; physician-patient relations; narrative; trust. 


\section{Resumo}

\section{Falhas na relação médico-paciente na esclerose múltipla}

Um bom relacionamento entre os médicos e os pacientes permite adiantar o diagnóstico de esclerose múltipla e conseguir adesão aos tratamentos. Este trabalho buscou compreender as dificuldades na relação entre os médicos e os pacientes com esta condição de saúde, através de pesquisa narrativa realizada no Instituto Neurológico da Colômbia, na cidade de Medellín, do qual participaram cinco pessoas com esclerose múltipla, dois cuidadores e dois profissionais que tratam a doença. Foram feitas entrevistas conversacionais em profundidade, visitaramse suas casas e foram avaliados seus prontuários médicos. Foi construída uma narração polifônica para cada participante, das quais foram extraídas algumas histórias que evidenciam as dificuldades na relaçáo médicopaciente. Esses relatos evidenciam a falta de confiança nas propostas terapêuticas dos médicos, bem como expressóes que demostram desprezo por seus pacientes. Além disso, demonstram o mal-estar pelas consultas tâo curtas e as dificuldades para chegar ao diagnóstico e expressá-lo apropriadamente. Esta situação aponta as dificuldades para realizar uma boa abordagem clínica, que pode afetar os acordos entre médicos e pacientes sobre os melhores procedimentos terapéuticos a serem adotados.

> Palavras-chave: esclerose múltipla; relações médicopaciente; narrativa; confiança. 